\title{
Communications
}

\section{Examen de spécialiste}

\section{Examen pour l'obtention de l'attestation de formation complémentaire en phlébologie}

Date: Lundi, 13 septembre 2010

Lieu: Bâle. Lors du 15e Congrès Tripartite des Sociétés suisse, allemande et autrichienne $\mathrm{d}^{\prime}$ Angiologie et du $11^{\mathrm{e}}$ Congrès de l'Union des Sociétés Suisses des Maladies Vasculaires

Délai d'inscription: le $1^{\text {er }}$ août 2010

Inscription: Prof. Dr méd. Jürg Hafner, USZ, Clinique de dermatologie, 8091 Zurich, tél. 04425525 60, fax 04425544 03, E-Mail: juerg.hafner@usz.ch

Informations: voir sous www.uvs.ch, lien AFC phlébologie

\section{Swiss Insurance Medicine SIM}

\section{Première certification d'experts} en Suisse romande

Pour la première fois, des certificats $\mathrm{d}^{\prime}$ «expert médical SIM» ont aussi été décernés en Suisse romande le 23 avril dernier. Dans une ambiance festive et en présence du vice-président de la FMH, le Dr Ignazio Cassis, et du directeur de la formation médicale, le Prof. Tissot, le certificat a été remis à 87 médecins à Lausanne. La SIM félicite vivement les nouveaux détenteurs et les nouvelles détentrices du certificat et se réjouit de voir enfin arriver cette extension à la Suisse romande. Désormais, 460 personnes sont porteuses de ce titre dans l'ensemble de la Suisse. Près de 200 autres personnes s'y ajouteront encore cette année en Suisse alémanique, si bien qu'il y aura déjà un nombre considérable d'experts et d'expertes certifié(e)s d'ici la fin de l'année. Les cours seront bien entendu poursuivis dans toutes les parties du pays et des journées thématiques permettront de continuer à améliorer la qualité. Il est possible de s'inscrire sous www.swiss-insurance-medicine.ch

\section{Stiftung Patientensicherheit}

Erfassung der Arzneimittel- und Medikationssicherheit in Schweizer Spitälern «Drug Event Monitoring DEM-AMS»

Untersuchungen zeigen, dass 30-50\% aller Behandlungsfehler Medikationsfehler sind. Mit einem nationalen Projekt nehmen sich die Stiftung für Patientensicherheit, die Stiftung für Arzneimittelsicherheit und das Schweizerische Heilmittelinstitut Swissmedic dieser Thematik an. Punktuelle Untersuchungen der Schweizerischen Stiftung für Arzneimittelsicherheit sowie internationale Publikationen zeigen, dass bis zu 7\% der Spitaleinweisungen aufgrund Arzneimittel-assoziierter Probleme oder wegen Medikationsfehlern erfolgen und mindestens $7,5 \%$ aller Spitalpatienten von unerwünschten Arzneimittelwirkungen oder Medikationsfehlern betroffen sind. Systematische Daten zur Situation in der Schweiz sind aber nur spärlich vorhanden. Zur Verbesserung der Datenlage starteten Swissmedic, die Stiftung für Patientensicherheit und die Stiftung für Arzneimittelsicherheit gemeinsam das Projekt «Drug Event Monitoring - Arzneimittelund Medikationssicherheit - DEM-AMS».

Die Ziele des Projektes sind:

- Entwicklung von Kennzahlen zur Arzneimittel- und Medikationssicherheit (Indikatoren, Aussagen zur Häufigkeit, pharmakoepidemiologische Daten);

- Erhebung und Testung der Kennzahlen in vorerst 3 Spitälern/Spitalnetzen, danach Ausweitung des Monitorings;

- Ableitung des Handlungsbedarfs für die Verbesserung der Arzneimittel- und Medikationssicherheit.

Die Anschubfinanzierung erfolgt durch Swissmedic und die Stiftung für Arzneimittelsicherheit. Die Projektleitung liegt bei der Stiftung für Patientensicherheit. In der Aufbauphase ist vorgesehen, mit drei Spitälern bzw. Spitalnetzen aus verschiedenen Sprachregionen der Schweiz zusammenzuarbeiten. Die Spitäler werden mit Ihrem Know-how von Anfang an in den Projektaufbau einbezogen und führen die Datenerfassung in der Pilotphase durch. Das Projekt wird berufsgruppenübergreifend geführt. Die Expertensicht und die Sicht der Patienten werden berücksichtigt. Ziel ist der Patientennutzen.
Das Projekt wurde im Januar 2010 gestartet. Bis Herbst 2010 werden fundierte Grundlagen entwickelt. Ab Herbst 2010 finden die ersten Datenerhebungen statt. Mit ersten Auswertungen ist im Frühjahr 2011 zu rechnen.

Weitere Informationen: Carla Meyer-Massetti, Drug Event Monitoring, Stiftung für Patientensicherheit, Asylstrasse 77, CH-8032 Zürich, Tel. 04324376 28, E-Mail: meyer@patienten sicherheit.ch; www.patientensicherheit.ch

\section{Schweizerische Fachgesellschaft ADHS}

\section{Neuerungen im Vorstand}

An der diesjährigen Hauptversammlung der Schweizerischen Fachgesellschaft ADHS beendeten Dr. med. Doris Ryffel-Rawak und Dr. med. Meinrad Ryffel ihre Vorstandstätigkeit. Doris Ryffel hat als Psychiaterin die Diagnose ADHS bei Erwachsenen unter ihren Fachkollegen bekannt gemacht. Ihre Bücher zum Thema dienen einem besseren Verständnis von ADHS bei Ärzten, Patienten und Angehörigen. Meinrad Ryffel setzte sich über Jahrzehnte für die Anliegen ADHS-betroffener Kinder und deren Familien ein. Das Ärzteehepaar war denn auch Mitinitiant der im Jahr 2005 gegründeten Fachgesellschaft ADHS, der heute bereits über 200 Fachpersonen angehören. Zum gleichen Zeitpunkt trat auch Prof. Dr. rer. nat. Rolf-Dieter Stieglitz aus dem Vorstand zurück. Er hatte seine Tätigkeit von Anfang an auf eine Amtsperiode begrenzt, Ziel war der Aufbau der Fachgesellschaft.

Neu wurden Dr. med. Martin Markarian, Kinder- und Jugendpsychiater aus Gland, Dr. med. Roland Kägi, Kinderarzt aus Zürich, und Dr. med. Hans-Rudolf Stricker, Facharzt Allgemeinmedizin und Verhaltenstherapeut aus Zürich, in den Vorstand berufen. Das Co-Präsidium teilen sich Prof. Dr. med. Dominique Eich, Fachärztin für Psychiatrie und Psychotherapie aus Zürich, und Dr. phil. Markus Stucki, Fachpsychologe für Psychotherapie aus Boll. Die bisherigen Vorstandsmitglieder Dr. med. Jürg Grossenbacher und Ursula Ammann, ADHS-Coach, wurden in ihrem Amt bestätigt. Weitere Informationen unter www.sfg-adhs.ch 